\title{
The COVID-19 Pandemic Impact on Patients with Obsessive Compulsive Disorder
}

\section{Opinion}

Volume 1 Issue 1- 2020

\begin{abstract}
Author Details
Hani Raoul Khouzam ${ }^{1,2}$

${ }^{1}$ Department of Mental Health and Psychiatry Services, PTSD treatment program and General Mental Health Clinic, VA Northern California Health Care System (VANCHCS), Sacramento VA Medical Center, Mather, USA

${ }^{2}$ Department of Psychiatry and Medicine, UC Davis Medical School, USA

*Corresponding author

Dr. Hani Raoul Khouzam, Clinical Professor of Psychiatry and Medicine, UC Davis Medical School, Sacramento, California, USA
\end{abstract}

Article History

Received: October 13, 2020 Accepted: November 25, 2020 Published: November 30, 2020

\begin{abstract}
The COVID-19 pandemic creates challenges for individuals with preexisting mental health conditions, such as those with depression, phobias, generalized anxiety, and obsessive-compulsive disorders. Patients with these preexisting disorders could experience increased fears about danger of contamination, and high level of distress. This report focuses on the diagnosis and the general treatment of obsessive-compulsive disorder and then describes specific interventions to prevent its clinical deterioration due to COVID-19 global pandemic.
\end{abstract}

Keywords: COVID-19; OCD; Cognitive behavioral therapy; Psychotherapy; Psychopharmacology; Treatment; Guidelines

\section{Introduction}

Preventive measures that are aimed at slowing the spread of COVID-19 such as the constant sanitizing and hand washing, could aggravate and worsen symptoms in patients suffering from obsessive compulsive disorder (OCD). Patients with OCD experience ongoing difficulties with controlling repetitive behaviors involving preoccupation with cleanness and indulgence in constant washing, cleaning, and cleansing. Other practices such as frequent selfmonitoring of temperature to check for fever; and repeated attempts to swallow saliva to check for throat pain as a symptom of COVID-19 could also occur. Handwashing being an anchor precaution to prevent COVID-19 transmission adds further to the misery of a known washer OCD patient. These repetitive behaviors are usually time consuming and detrimental to daily interpersonal, educational and vocational functioning. Exacerbation of OCD has been previously reported during other infectious outbreaks, such as Severe Acute Respiratory Syndrome (SARS), Middle East Respiratory Syndrome (MERS), and Influenza [1]. Consequently, recent studies on COVID-19 have also reported the need to maintain clinical vigilance to monitor potential relapse of OCD symptoms and to initiate early intervention to prevent exacerbation and worsening of patients' ability to maintain their already achieved stability $[2,3]$.

\section{OCD and its Global Burden}

It is estimated that between 2 and 3 million people are suffering from OCD in the United States and about one in fifty people have had symptoms of OCD at some point in their lives, with $1 \%$ suffering within the last year. OCD afflicts people of all races, faiths, nationalities, and ethnic groups. OCD causes great suffering to patients and their families, as up to 10 hours per day may be devoted to performing rituals. OCD has been classified by the World Health Organization (WHO) as the sixth and one of most disabling psychiatric disorder with lifetime prevalence of $1.9-3.3 \%$ [4]. Among the wide cluster of OCD symptoms, fear of dirt, feeling of being contaminated with excessive washing and cleaning are the most common manifestation of this disorder $[5,6]$. In that context the precautionary measures of vigilance in washing and cleaning that are instituted to prevent COVID-19, pose a disproportionate challenge for patients with OCD whose mental stability have been achieved through abstaining from practicing excessive washing and cleaning.

\section{OCD and its Recognition}

The Diagnostic and Statistical Manual of Mental Disorders, Fifth Edition (DSM-5) reclassified OCD not as an anxiety disorder and included it under a new category of "Obsessive-Compulsive and 
Related Disorders" which also include Body Dysmorphic Disorder ,Hoarding Disorder, Trichotillomania and Excoriation (Skin Picking) Disorder [7].

\section{Obsessions}

These are usually described as recurrent and persistent thoughts, impulses, or images that are intrusive and cause marked distress and they do not reflect excessive worries about real-life problems. Attempts are constantly exerted to ignore, suppress, or neutralize these thoughts, impulses, or images to no avail. The obsessional thoughts, impulses, or images are identified by patients as being not justifiable and unwarranted. The OCD Obsessions are not a manifestation of a delusional or a psychotic disorder.

\section{Compulsions}

These are defined as repetitive behaviors or mental acts that the person feels driven to perform to prevent or reduce the distress caused by the obsessions. Many individuals with OCD experience both obsessions and compulsions. Some patients with OCD do not experience compulsions and are often referred to as "Pure O's", or "Pure Obsessionals" [8].

The exact causes of OCD are unknown with environmental and genetic factors believed to play a role in its development $[9,10]$. There is also an increased incidence in identical than non-identical twins [11]. Some cases have been documented to occur following infections [12]. Risk factors include a history of child abuse or stress-inducing event [7]. The symptoms of OCD are not the result of another psychiatric disorder, a medical condition or substance use [2]. Rating scales such as the YaleBrown Obsessive Compulsive Scale (Y-BOCS) can be used to assess OCD severity [13]. Other disorders with similar OCD symptoms include anxiety disorder, major depressive disorder, eating disorders, tic disorders, and obsessive-compulsive personality disorder [7]. OCD is associated with reduced quality of life as well as high levels of social and occupational impairment. Impairment occurs across many different domains of life and is associated with symptoms severity [13]. Impairment can be caused by the time spent obsessing and then performing the compulsions. Avoidance of situations that can trigger obsessions or compulsions can also severely restrict functioning. In addition, specific symptoms can create specific obstacles. For example, obsessions about harm can make relationships with family and friends feel hazardous; the result can be avoidance of these relationships. Obsessions about symmetry can derail the timely completion of school or work projects because the project never feels "just right", potentially resulting in school failure or job loss. Health consequences can also occur. For example, individuals with contamination concerns may avoid doctors' offices and due to fears of exposure to germs or develop serious dermatological complications such as skin lesions due to excessive washing. Sometimes the symptoms of the disorder interfere with recommended if the medications are considered contaminated. When the disorder starts in childhood or adolescence, individuals may experience developmental difficulties. Adolescents may avoid socializing with peers; and young adults may struggle when they leave home to live independently. These self-imposed social restrictions could result in only few significant relationships outside the family and could lead to a lack of autonomy and financial independence. In addition, some patients with OCD may attempt to impose rules and prohibitions on family members because of their obsessions and fear of contamination leading to family conflicts and dysfunctional relationships [14].

\section{OCD Comorbidity}

Patients with OCD often have other lifetime co-occurring psychiatric disorders such obsessive-compulsive personality disorder, major depressive disorder, bipolar disorder, generalized anxiety disorder, posttraumatic stress disorder, anorexia nervosa, social anxiety disorder, bulimia nervosa, Tourette syndrome, autism spectrum disorder, attention deficit hyperactivity disorder, excoriation (Skin Picking) disorder, body dysmorphic disorder and trichotillomania [15]. More than $50 \%$ of patients with OCD would experience suicidal tendencies, and up to $15 \%$ would attempt suicide [16]. Depression, anxiety and prior suicide attempts increase the risk of future suicide attempts [17]. A triad of OCD, tic disorder, and attention deficit hyperactivity disorder have also co-occurred in children [18]. To achieve a good treatment prognosis patient with OCD would also need to have appropriate and timely treatment of their comorbid conditions.

\section{OCD and its Treatment}

Several treatment interventions have been effective in reducing the symptoms of OCD. Treatment usually combine psychotherapy and psychopharmacology.

\section{Psychotherapy}

Cognitive Behavior Therapy (CBT) is considered the "gold standard," or the treatment of choice, for OCD [19]. It implements two evidencebased techniques - Exposure and Response Prevention therapy (ERP) and Cognitive Therapy (CT). The first step in ERP instruct patients to provide their psychotherapist with a detailed description of the obsessions and compulsions, which are then ranked from the least distressing to the most difficult. Then, beginning with the milder obsessions, therapists explore "exposures," or challenges, and situations that would trigger the obsessions. During these exposures, patients would avoid performing the compulsive behaviors ("response prevention") for increasingly longer periods of time. Through repeated exposures, patients then realize that when the compulsions are avoided, initially there is a temporary increase in the level of distress which peaks and then subsides. When it is practically impossible to create the actual situations that trigger the compulsions, the therapists then would use imagined exposures, visualizations and recordings that could raise distress levels to allow for the practice of ERP exercises. The therapeutic exposures are repeated over time, thus resulting to decreased levels of associated distress until it gradually and eventually fades entirely. More challenging exposures are then introduced in subsequent therapy sessions until they, too, become manageable. Effective ERP leads to "habituation," and recognizing that no catastrophic outcomes would occur if the compulsive responses and rituals are not performed. Cognitive therapy (CT), which is the second component of $\mathrm{CBT}$, is based on identifying and modifying patterns of thought that cause anxiety, distress, or compulsive behaviors. In CT patients spend time in searching for the thoughts and the obsessions that trigger the undesirable behaviors or compulsions and then identifying them as "faulty" or "unrealistic" thoughts .Then gradually learning to recognize and confronting the obsessions and responding to them in alternative and non - compulsive rituals. Strategies such as mindfulness, learning to separate oneself from the obsessions, and analyzing the realistic chances or probability of a catastrophe's occurrence are also components of CT. Symptoms of OCD interfere with social-interpersonal relationships and daily functioning and create challenges for family members, caregivers, teachers, co-workers and friends, who may experience frustration, confusion and helplessness . Support groups and parent management training (PMT) could lessen the interpersonal conflicts improve treatment outcomes [20]. Most CBT therapy sessions are conducted in outpatient settings weekly with "homework" consisting of daily exposures to be completed between therapy sessions. In severe cases, some patients may require more frequent sessions or even specialized residential treatment [21]. Group CBT therapy sessions for adults [22], and group cognitive-behavioral family-based therapy (CBFT) for childhood OCD symptoms can also be alternative therapeutic options [23].

\section{Psychopharmacology}

Current evidence continues to support the use of selective serotonin reuptake inhibitors(SSRI's), as first-line pharmacologic interventions 
for OCD [13,24]; however, more recent data support the adjunctive use of neuroleptics, deep-brain stimulation, and neurosurgical ablation for treatment-resistant OCD [24]. Preliminary data suggest safety of other agents such as riluzole, ketamine, memantine, $\mathrm{N}$-acetylcysteine, lamotrigine, levetiracetam, celecoxib, and ondansetron either in combination with SSRI's or as monotherapy in the treatment of OCD, have not yet been established [14,24].

\section{The Effects of COVID-19 on OCD}

COVID-19 which is caused by SARS-CoV-2 viral infection, is considered a disaster not only due to its psychosocial impact but it also to its direct effects on the brain. The complexity of COVID-19's pathology and its impact on the brain requires appropriate screening that has to go beyond the psychosocial impact, considering how the combination of stress and neuroinflammation affects the brain [25]. As a result ,a basic "NeuroCovid" classification scheme have been recently proposed to integrate the short-term challenges and the longterm sequalae of COVID-19 in the context of its effects on depression, OCD, insomnia, cognitive decline, accelerated aging, Parkinson's disease, and Alzheimer's disease [26]. To address OCD as a potential complication of NeuroCovid, clinical multidisciplinary treatment approach would be required to treat and prevent all of Sars-Cov-2 possible mental health sequelae [25].

The COVID-19 global pandemic is affecting some patients with OCD with several reports indicating recurrence or increased intensity of symptoms and overwhelming concerns in several domains such as those that are summarized in the following: Table 1.

Table 1: Difference between Obsessions and Compulsions.

\begin{tabular}{|c|c|}
\hline Obsessions & Compulsions \\
\hline Fears of personal contamination & Increased frequency of washing and cleaning \\
\hline Fears of infecting others & Repetitive checking of own body temperature throughout the day every day \\
\hline $\begin{array}{c}\text { Intrusive thoughts about accidental close contact with } \\
\text { significant other }\end{array}$ & $\begin{array}{c}\text { Constant vigilant monitoring of one's behavior, even while maintain social } \\
\text { distancing }\end{array}$ \\
\hline Preoccupation about being unhealthy & Excessive measures of monitoring food intake and daily physical exercises \\
\hline Doubting own mental stability & Frequent phone calls and virtual appointments with mental health professionals \\
and agencies
\end{tabular}

\section{OCD and its Management during the COVID-19 Pandemic}

the International College of Obsessive Compulsive Spectrum Disorders (ICOCS) and the Obsessive-Compulsive and Related Disorders Research Network (OCRN) of the European College of Neuropsychopharmacology have suggested certain clinical guidelines for the management of OCD under COVID-19 [27], which are summarized in the following section.

I. Despite social distancing and limited face to face contact, an approach based on compassion, and reflection of calm could be implemented, by utilizing telemedicine to include frequent telephone or video calls if clinically indicated to minimize and prevent symptoms exacerbation.

II. Confirming the diagnosis of OCD, to assure that the presenting symptoms are not related to other Obsessive-Compulsive and Related Disorders which include Body Dysmorphic Disorder, Hoarding Disorder, Trichotillomania and Excoriation (Skin Picking) Disorder [7], or hypochondriasis [28]. If OCD is not the principal diagnosis for clinical attention, other guidelines regarding mental health and psychosocial considerations during the COVID-19 outbreak should be considered [29].

III. Assessing suicidal risk. As a disorder, OCD has a high suicide risk, especially when it co-occurs with depression, bipolar disorder, impulse control disorders, substance use disorders, personality disorders and eating disorders $[16,17,30]$. In addition, COVID-19 associated factors such as a recent increase in OCD severity, infection in a family member and the accumulating effects of quarantine and distressing social isolation could potentially increase the suicide risk. If these factors are present, patients with OCD, should be specifically assessed for eminent suicide risk [31].

IV. Psychoeducation that focus on providing accurate and balanced information about the known risks and impact of COVID-19 on physical and mental wellness. The need for physical distancing should be empathized as a preventive measure against getting infected with ongoing practice of learned CBT techniques, daily exercise and structured activities to prevent recurrence of obsessions and compulsions related to cleaning, washing and cleansing.

V. If significant exacerbation of OCD symptoms occur due to excessive Internet usage and news consumption to seek the latest COVID-19 updates ; then it is of paramount importance to limit the number of times of checking the information and to also restrict access to the many sources that are providing the information. Limiting access to two or three trusted websites such as the World Health Organization (WHO) and Centers for Disease Control (CDC), and avoiding endless hours of watching television and online media sources, to prevent further worsening of OCD symptoms [32].

\section{The Importance of Faith and Spirituality}

Spiritual leaders of all faiths may need to develop an alliance and coordinated cooperation with mental health professionals in an effort to develop crisis communication, mutual aid networks and other programs. These Programs could be aimed at protecting psychosocial and spiritual wellbeing of all patients with pre-existing psychiatric conditions who may experience exacerbation of their symptoms due to the COVID-19 pandemic even in those who have not been infected by this potentially deadly virus. Religious leaders and clergy who volunteered during hurricanes, floods and fires and who have expertise in managing the mental health consequences of these disasters could also serve as resources during this pandemic to strengthen the faith in OCD patients who may have stopped practicing their religious and spiritual beliefs due quarantine ,boredom, loneliness, or anger toward God. In many biologically induced disasters, themes of fear, uncertainty, and stigmatization are common and may create barriers to appropriate medical and mental health interventions [33,34]. Based on experiences from past serious global infectious disease outbreaks and viral epidemics, the reliance on faith and religious beliefs could mobilize inner strengths and move patients beyond fears, doubts, and hopelessness and instill new meaning to life's purposes. During these unprecedented times of confronting the many unknown impacts of COVID-19 on the human mind, body and spirit, the rekindling of faith and spiritual beliefs could offer an opportunity for the revitalization of the boundless strengths of the human soul. 


\section{Conclusion}

Many patients with OCD are at risk of experiencing symptoms exacerbation with heightened levels of distress due the COVID-19 pandemic .Certain measures to maintain the ongoing stability of patients need to be implemented to provide effective care for patients with OCD .The best available treatments for most patients are likely to include providing evidence-based CBT and pharmacotherapy. Addressing the impact of the pandemic on the expression of OCD symptoms would provide patients with support and sustain their ongoing pre-COVID-19 mental stability. Suggested guidelines could also equip clinicians with the necessary tools that are needed to implement effective interventions during this disastrous and unprecedented global health crisis.

\section{Acknowledgment}

Sincere gratitude and thankfulness to my wife Lynn and children, Andrea and her husband Nic, and their daughter Abigail, Andrew and Adam, my sisters Hoda and Héla, and my brother Hadi for their support and encouragement.

\section{Conflict of Interest}

Author has no conflict of interest.

\section{References}

1. Banerjee DD (2020) The other side of COVID-19: Impact on obsessive compulsive disorder (OCD) and hoarding. Psychiatry Res 288: 112966.

2. Fontenelle LF, Miguel EC (2020) The impact of COVID-19 in the diagnosis and treatment of obsessive-compulsive disorder. Depression Anxiety 37(6): 510-511.

3. Ho CS, Chee CY, Ho RC (2020) Mental health strategies to combat the psychological impact of COVID-19 beyond paranoia and panic. Ann Acad Med Singap 49: 156-159.

4. WHO (2004) World Health Organization Update.

5. Akhtar S, Wig NN, Varma VK (1975) A phenomenological analysis of symptoms in obsessive compulsive neurosis. Br J Psychiatry 127: 342348 .

6. Lochner C, Hemmings SM, Kinnear CJ (2008) Cluster analysis of obsessive-compulsive symptomatology: identifying obsessivecompulsive disorder subtypes. Isr J Psychiatry Relat Sci 45(3): 164-176.

7. (2013) Diagnostic and statistical manual of mental disorders : DSM-5 ( $5^{\text {th }}$ Edn.). Washington: American Psychiatric Publishing. 2013 237-242.

8. Fergus TA (2013) Thought control moderates the relation between autogenous intrusions and the severity of obsessional symptoms: Further support for the autogenous-reactive model of obsessions .Journal of Obsessive-Compulsive and Related Disorders 2(1): 9-13.

9. Bloch MH, Pittenger C (2010) The Genetics of Obsessive-Compulsive Disorder. Curr Psychiatry Rev 6(2): 91-103.

10. Pauls DL, Abramovitch A, Rauch SL, Geller DA (2014) Obsessivecompulsive disorder: an integrative genetic and neurobiological perspective. Nat Rev Neurosci 15(6): 410-424.

11. van Grootheest DS, Cath DC, Beekman AT, Boomsma DI (2005) Twin studies on obsessive-compulsive disorder: a review. Twin Res Hum Genet 8(5): 450-458.

12. Chiarello F, Spitoni S, Hollander E (2017) An expert opinion on PANDAS/PANS: highlights and controversies. Int J Psychiatry Clin Pract 21(2): 91-98.

13. Khouzam HR (1999) Obsessive-Compulsive Disorder. What to do if you Recognize Baffling Behavior. Postgraduate Medicine 106(7): 133-141.

14. Khouzam HR (2015) Levetiracetam Treatment of Refractory ObsessiveCompulsive Disorder. Ann Psychiatry Ment Health 3(6): 1045.

15. Dell'Osso B, Pallanti S, Stein DJ (2014) Comorbidity in obsessivecompulsive disorder (OCD): a report from the International College of Obsessive-Compulsive Spectrum Disorders (ICOCS). Compr Psychiatry 55(7): 1513-1519.
16. Breet E, Kidd M, McGregor NW (2019) Suicide ideation and attempts in obsessive-compulsive disorder. Ann Clin Psychiatry 31(3): 192-199.

17. Albert U, De Ronchi D, Maina G, Pompili M (2019) Suicide Risk in Obsessive-Compulsive Disorder and Exploration of Risk Factors: A Systematic Review. CurrNeuropharmacol 17(8): 681-696.

18. Martino D, Ganos C, Pringsheim TM (2017) Tourette Syndrome and Chronic Tic Disorders: The Clinical Spectrum Beyond Tics. Int Rev Neurobiol 134: 1461-1490.

19. McKay D, Sookman D, Neziroglu F (2015) Efficacy of cognitivebehavioral therapy for obsessive-compulsive disorder. Psychiatry Res 225(3): 236-246.

20. Sukhodolsky DG, Gorman BS, Scahill L (2013) Exposure and response prevention with or without parent management training for children with obsessive-compulsive disorder complicated by disruptive behavior: a multiple-baseline across-responses design study. J Anxiety Disord 27(3): 298-305.

21. Björgvinsson T, Hart AJ, Wetterneck C (2013) Outcomes of specialized residential treatment for adults with obsessive-compulsive disorder. J PsychiatrPract 19(5): 429-437.

22. Papageorgiou C, Carlile K, Thorgaard S (2018) Group CognitiveBehavior Therapy or Group Metacognitive Therapy for ObsessiveCompulsive Disorder? Benchmarking and Comparative Effectiveness in a Routine Clinical Service. Front Psychol.

23. O'Leary EM, Barrett P, Fjermestad KW (2019) Cognitive-behavioral family treatment for childhood obsessive-compulsive disorder: a 7-year follow-up study. J Anxiety Disord 23(7): 973-978.

24. Hirschtritt ME, Bloch MH, Mathews CA (2017) Obsessive-Compulsive Disorder: Advances in Diagnosis and Treatment. JAMA 317(13): 13581367.

25. Pallanti S, Grassi E, Makris N,et al. Neurocovid-19: A clinical neuroscience-based approach to reduce SARS-CoV-2 related mental health sequelae. J Psychiatr Res. 2020 Aug 15;130:215-217

26. Fotuhi M, Mian A, Meysami S, Raji CA. Neurobiology of COVID-19. J Alzheimers Dis. 2020;76(1):3-19.

27. Fineberg NA, Van Ameringen M, Drummond L, et al. How to manage obsessive-compulsive disorder (OCD) under COVID-19: A clinician's guide from the International College of Obsessive Compulsive Spectrum Disorders (ICOCS) and the Obsessive-Compulsive and Related Disorders Research Network (OCRN) of the European College of Neuropsychopharmacology. ComprPsychiatry . 2020 Jul;100:152174. doi: 10.1016/j.comppsych.2020.152174.

28. World Health Organization ICD-11 - Mortality and Morbidity Statistics. Geneva. April 2019. https://icd.who.int/browse11/1-m/en.

29. World Health Organization Mental health and psychosocial considerations during the COVID-19 outbreak. https://www.who.int/ docs/default-source/coronaviruse/mental-health-considerations.

30. Pellegrini L, Maietti E, Rucci P, et al. Suicide attempts and suicidal ideation in patients with obsessive-compulsive disorder: a systematic review and meta-analysis. J Affect Disord. 2020 Nov 1;276:1001-1021. doi: 10.1016/j.jad.2020.07.115.

31. Posner K, Brown GK, Stanley B, et al. The Columbia-Suicide Severity Rating Scale: initial validity and internal consistency findings from three multisite studies with adolescents and adults. Am J Psychiatry. 2011;168(12):1266-1277.

32. Adhanom Ghebreyesus T. Addressing mental health needs: an integral part of COVID-19 response. World Psychiatry (2020) 19(2):129-30. 10.1002/wps.20768.

33. Xiang YT, Yang Y, Li W, et al. Timely mental health care for the 2019 novel coronavirus outbreak is urgently needed. Lancet Psychiatry. 2020;7(3):228-229. doi: 10.1016/S2215-0366(20)30046-8.

34. Smith BJ, Lim MH. How the COVID-19 pandemic is focusing attention on loneliness and social isolation. Public Health Res Pract. 2020 Jun 30;30(2):3022008. doi: 10.17061/phrp3022008. 